\title{
Two-dimensional Stokes flow with suction and small surface tension
}

\author{
L.J. CUMMINGS ${ }^{1}$ and S.D. HOWISON ${ }^{2}$ \\ 1 Labo. de Phys. Stat. de l'E.N.S., 24 rue Lhomond, 75231 Paris Cedex 05, France \\ 2 Mathematical Institute, 24-29 St Giles', Oxford OX1 3LB, England.
}

(Received 10 April 2006)

\begin{abstract}
In this article the complex variable theory of two-dimensional Stokes flow as developed by Richardson [23], and modified by Howison \& Richardson [17], is reviewed. The analysis of [17] is extended to a new solution, which uses a cubic polynomial conformal mapping (with real coefficients) from the unit disc onto the fluid domain. An apparent "stability paradox" (where two equivalent flow geometries are found, one of which is 'stable' and the other unstable) is resolved by allowing the coefficients to take complex values.
\end{abstract}

\section{Introduction}

The problem of two-dimensional slow viscous flow (Stokes flow) with a free boundary under surface tension has been the subject of much research in the last few years. ${ }^{1}$ It has long been known that many exact steady solutions exist, for both viscous "blobs" and for bubbles in unbounded fluid domains (for instance, Richardson [21,22], Garabedian [9], Buckmaster [2]). The recent interest stems from the remarkable discovery that many exact time-dependent solutions to the problem can be found in closed form, using ideas from complex variable theory [11-13,23].

More recently still, Howison \& Richardson [17] considered time-evolving problems incorporating a driving mechanism (a single point sink) at some finite point within the (bounded) fluid domain, and Tanveer \& Vasconcelos [26] solved for similar problems but with bubbles evolving in time under the action of some prescribed flow at infinity. Stokes flows with singularities have been considered before, but to the authors' knowledge, all such treatments have been for the steady problem; see for instance $[1,19]$, where the flow is sustained by a vortex dipole singularity. When we allow such singularities in the unsteady problem, the possibility is raised of considering the (much easier) zero surface tension (ZST) problem.

This problem admits very many simple exact solutions, and in physical situations where the relevant dimensionless surface tension parameter $T$ (which is a measure of the effects of surface tension versus those of the driving mechanism) is small, these may provide a good approximation to the actual solution. However, the ZST problem has the drawback that its solutions can undergo finite time blow-up, with curvature singularities (often, but not always, cusps) appearing in the free boundary - this is the case for the point-sink driven problems considered in [17] ( $c f$. the ZST Hele-Shaw problem with suction, also discussed in [17]). The approximate ZST boundary condition becomes invalid as such a curvature singularity develops. Nevertheless, for small values of the surface tension parameter, the evolution of the ZST solution prior to blow-up gives a good approximation to the solution with positive surface tension. In such problems, it seems reasonable to consider the evolution in two parts, assuming "ZST theory" until we near blow-up time, and only then introducing surface tension effects. Experimental evidence, showing that very highly-

1 Stokes flow is of practical importance in many industrial processes, some of which are listed in [26], and discussed in references therein. 
curved interfaces can be sustained in driven flows, supports this idea [2,19]. In [17] the authors take this idea to its logical conclusion, considering the limit $T=0^{+}$. We emphasise that the difficulties encountered (due to the singular limit $T \rightarrow 0^{+}$) when attempting to regularise the ZST Hele-Shaw problem by introducing small positive surface tension, do not arise for Stokes flow. We also point out that for Stokes flow, before singularity formation the ZST problem is identical to the zero-surface-tension limit of the problem with $T>0$. This question remains unresolved at a rigorous level for the Hele-Shaw problem, although [25] suggests that in this case the limiting regularised problem can differ from the unregularised problem at an $O(1)$ time before that of singularity formation.

This paper explores this concept further, applying it to analyse a more complicated example. Our initial "simplest case" analysis, in $\S 5$, throws up an apparent paradox, the resolution of which (in $§ 5.2$ ) reveals a surprisingly rich solution structure. The results we obtain are of interest both in the general study of (experimentally observable) cusp formation in viscous flow $[1,2,19,20]$, and also in the wider mathematical context of conformal mapping techniques applied to free boundary problems.

\section{Complex variable theory}

We begin by outlining the theory as developed in $[17,23]$. The notation we employ is largely the same as those papers. It is convenient to work with the streamfunction $\psi(x, y, t)$, which in two-dimensional slow viscous flow satisfies the biharmonic equation

$$
\nabla^{4} \psi=0
$$

The fluid is assumed to occupy some simply-connected domain $\Omega(t)$ in $\mathbb{R}^{2}$, which we can consider as a subset of $\mathbb{C}$ if we write $z=x+i y$.

As well as the kinematic boundary condition (equating the velocity of the fluid at the free boundary, in the direction of the outward normal, to the velocity of the free boundary itself in that direction) there are two stress boundary conditions,

$$
\sigma_{i j} n_{j}=-T \kappa n_{i} \quad i=1,2, \quad \text { on } \partial \Omega(t) .
$$

Here, $\sigma_{i j}$ denotes the usual Newtonian stress tensor, $\mathbf{n}=\left(n_{i}\right)$ denotes the outward normal to $\partial \Omega(t), T$ is a surface tension coefficient, and $\kappa$ is the curvature of the free boundary.

Using the Goursat representation of biharmonic functions $\psi$, together with its biharmonic conjugate the Airy stress function $\mathcal{A}$, may be expressed in the form

$$
\mathcal{A}+i \psi=\mathcal{W}(z, \bar{z}, t)=-(\bar{z} \phi(z, t)+\chi(z, t)),
$$

for functions $\phi(z, t), \chi(z, t)$ analytic on the flow domain except at driving singularities. In this paper we consider only flows driven by a point sink (or source) of strength $Q$ at the origin, where $\phi$ and $\chi$ have the local behaviour

$$
\phi(z, t)=O(1), \quad \chi^{\prime}(z, t)=\frac{Q}{2 \pi z}+O(1), \quad \text { as } z \rightarrow 0 ;
$$

if $Q<0$ we have a point source. (We use ' for $\partial / \partial z$ or $\partial / \partial \zeta$ without ambiguity.) All physical quantities of interest can be expressed in terms of the functions $\phi$ and $\chi$; for instance, the velocity field $(u, v)$ and the pressure field $p$ are given by

$$
u+i v=\phi(z, t)-z \overline{\phi^{\prime}(z, t)}-\overline{\chi^{\prime}(z, t)}, \quad p=-4 \mu \Re\left\{\phi^{\prime}(z, t)\right\},
$$

where $\mu$ is the viscosity of the fluid. In terms of these functions the condition (2.1) becomes

$$
\phi(z, t)+z \overline{\phi^{\prime}(z, t)}+\overline{\chi^{\prime}(z, t)}=\frac{i T}{2 \mu} \frac{d z}{d s} \quad \text { on } \partial \Omega(t) .
$$


We now introduce a time-dependent conformal map $f(\zeta, t)$ from the unit disc in $\zeta$-space onto the fluid domain. This is always possible by the Riemann mapping theorem, and the map is uniquely determined if we insist $f(0, t)=0, f^{\prime}(0, t)>0$ for all time. We define the functions ${ }^{2} \Phi(\zeta, t)$ and $\mathcal{X}(\zeta, t)$ on the unit disc by

$$
\Phi(\zeta, t)=\phi(f(\zeta, t), t), \quad \mathcal{X}(\zeta, t)=\chi(f(\zeta, t), t) .
$$

The boundary conditions (2.1) and the kinematic boundary condition may now be formulated in the $\zeta$-plane, as identities holding on $|\zeta|=1$. We refer to Richardson [23] for the details; briefly, when the (purely mathematical) condition $\Phi(0, t)=0$ is assumed, these identities are easily analytically continued, giving functional identities which hold globally in the $\zeta$-plane (equations (2.18) and (2.19) of [23]). ${ }^{3}$ These equations are most conveniently expressed as follows (see for example $[6,7])$ :

$$
\frac{\partial}{\partial t}\left(f^{\prime}(\zeta, t) \bar{f}(1 / \zeta, t)\right)+2 \mathcal{X}^{\prime}(\zeta, t)=\frac{T}{2 \mu} \frac{\partial}{\partial \zeta}\left(\zeta f^{\prime}(\zeta, t) \bar{f}(1 / \zeta, t) G_{+}(\zeta, t)\right),
$$

and

$$
2 \Phi(\zeta, t)-\frac{\partial f}{\partial t}(\zeta, t)+\frac{T}{2 \mu} G_{+}(\zeta, t) \zeta f^{\prime}(\zeta, t)=0
$$

where the function $G_{+}(\zeta, t)$ (analytic on $|\zeta| \leq 1$ ) is defined in terms of the conformal map via

$$
G_{+}(\zeta, t)=\frac{1}{2 \pi i} \oint_{|\tau|=1} \frac{1}{\left|f^{\prime}(\tau, t)\right|} \frac{\tau+\zeta}{\tau-\zeta} \frac{d \tau}{\tau} .
$$

Hence the solution procedure entails a search for conformal maps $f(\zeta, t)$ satisfying $(2.3)$ and (2.4), together with appropriate behaviour at the driving singularities. For the case of a single point sink at the origin this behaviour is

$$
\mathcal{X}(\zeta, t)=\frac{Q}{2 \pi} \log \zeta+O(1) \quad \text { as } \zeta \rightarrow 0
$$

$(\Phi(0, t)=0$ by assumption). Once one has postulated a suitable form for $f(\zeta, t)$, one can find a solution to the problem by matching the singular behaviour on either side of the equation (2.3). For our solution we shall use a slightly different approach however, based on results outlined in $\S 3$, which is particularly appropriate for polynomial mapping functions.

A crucial requirement of the theory (on both physical and mathematical grounds) is that the mapping function $f(\zeta, t)$ be univalent on the unit disc; solutions break down when this condition is violated. See [8] for a detailed discussion; loosely speaking, $f$ is required to be analytic and oneto-one, with nonvanishing derivative (conformal). If we assume an initially univalent map $f(\zeta, 0)$, then any such nonunivalency must approach $|\zeta|=1$ from without, hence nonunivalencies are first manifested on the boundary of the domain, in both $\zeta$-space and $z$-space (physical space). For instance, if the mapping becomes double-valued at some point on the unit circle, this corresponds to the fluid domain beginning to overlap itself, at which point the model must be modified to account for the change in topology. A zero in the derivative of $f$ on the unit circle corresponds to a cusp (or, exceptionally, a corner) in the free boundary, which we might expect to be smoothed off by the action of positive surface tension. We refer forward to Figure 2 for examples of selfoverlapping and cusped flow domains.

The conformal maps we shall use contain various time-dependent parameters, not all values of which give rise to a univalent map on $|\zeta| \leq 1$. In general there is a subset of the parameter space on

2 This notation differs from that of Richardson [23] since he uses $w(\zeta, t)$ rather than $f(\zeta, t)$ for the conformal map, and takes $X(\zeta, t)=\chi^{\prime}(w(\zeta, t), t)$, which may lead to confusion.

${ }^{3}$ Imposition of this condition can lead to solutions which do not conserve overall momentum (or equivalently, which have moving singularities within the flow). We refer to [7] or [17] for further discussion of this point. 
which the map is univalent on the unit disc, and to which we must restrict attention when solving problems. The idea of such a "univalency domain" (which we denote by $V$ ) in parameter space is an important concept in this paper, since one way to represent the solution is as a trajectory followed within $V$ as the parameters evolve in time. A trajectory reaching the boundary of $V$ is then associated with blow-up of the solution, by one of the means listed above. (See [18] for the application of similar ideas in the context of the Hele-Shaw free boundary problem.)

\section{Stokes flow "moments"}

Consider the quantities $C_{k}(t)$ defined (for integers $k \geq 0$ ) by

$$
C_{k}(t)=\iint_{\Omega(t)} \zeta(z, t)^{k} d x d y=\frac{1}{2 i} \int_{\partial \Omega(t)} \zeta(z, t)^{k} \bar{z} d z=\frac{1}{2 i} \int_{|\zeta|=1} \zeta^{k} f^{\prime}(\zeta, t) \bar{f}(1 / \zeta, t) d \zeta
$$

It follows from equation (2.3) (see [6] for the details; also [5] where similar ideas are applied) that with only the singularity (2.6) at the origin,

$$
\begin{aligned}
& \frac{d C_{0}(t)}{d t}=-Q \\
& \frac{d C_{k}(t)}{d t}=-\frac{k T}{2 \mu} \sum_{r=0}^{\infty} \frac{G_{+}^{(r)}(0, t)}{r !} C_{k+r}(t), \quad k \geq 1 .
\end{aligned}
$$

The quantity $C_{0}(t)$ is clearly the area of the fluid domain, so the $k=0$ equation just represents conservation of mass. Note that the ZST case is particularly simple, leading to an infinite set of conserved quantities.

For a general polynomial mapping function,

$$
f(\zeta, t)=\sum_{1}^{N} a_{r}(t) \zeta^{r}
$$

the $C_{k}(t)$ are readily evaluated from $(3.1)$ as

$$
C_{k}=\pi \sum_{n=1}^{N-k} n a_{n} \bar{a}_{n+k} \quad 0 \leq k \leq N-1,
$$

all other $C_{k}$ being identically zero (note that this shows that if $f(\zeta, 0)$ is a polynomial of degree $n$ then it must remain so for $t>0$ ). Hence we obtain a system of $N$ equations, (3.2) and (3.3), for the nonzero $C_{k}$, which are equivalent to a system of equations for the $N$ coefficients $a_{k}(t)$. In the ZST case these are algebraic, but the general NZST equations are a system of nonlinear ordinary differential equations.

\section{The limit of zero surface tension}

It is clear from the above that the ZST problem is very much simpler than the NZST one. Nonetheless, several time-dependent problems have been solved exactly using conformal mapping ideas similar to those outlined here, including:

(1) The coalescence under surface tension of two (equal or unequal) circular cylinders of fluid $[11,23]$;

(2) The coalescence under surface tension of a cylinder and a half-space of fluid [13];

(3) A limaçon evolving under the action of surface tension only [23];

(4) The evolution of domains described by polynomial maps of the form

$$
f(\zeta, t)=a(t)\left(\zeta-b(t) \zeta^{n} / n\right)
$$


for any integer $n \geq 2$, under the action of both surface tension and a point sink at the origin [17] ((3) above is a special case of this solution family, with $n=2$ and no suction);

(5) The evolution of bubbles in a shear flow with surface tension effects included [26];

(6) The evolution of expanding/contracting bubbles in otherwise quiescent flow with surface tension effects included [26].

The work that we are concerned with here is that of (4) above, due to Howison \& Richardson ( $[17]$; henceforth HR'95), since they include the effects of both surface tension and a driving mechanism; we thus give a short review of their work. They consider fluid domains $\Omega(t)$ driven by a single point sink of strength $Q>0$ at the origin, which are described by the family of mapping functions

$$
z=f(\zeta, t)=a(t)\left(\zeta-\frac{b(t)}{n} \zeta^{n}\right), \quad|\zeta| \leq 1,
$$

for $a(t), b(t)$ real and positive functions of time, and integers $n \geq 2$. The maps (4.1) are univalent on the unit disc only if $b(t)<1$, with $(n-1)$ inward-pointing $3 / 2$-power cusps forming simultaneously on $\partial \Omega(t)$ if $b(t)=1$.

We use the results of $\S 3$. By (3.5) the only nonzero moments are $C_{0}$ and $C_{n-1}$. The evolution of these quantities is given by (3.2) and (3.3), hence we must calculate $G_{+}(0)$ using (2.5). This is straightforward, leading to the evolution equations

$$
\begin{gathered}
\frac{d S}{d t}=\frac{d}{d t}\left[\pi a^{2}\left(1+\frac{b^{2}}{n}\right)\right]=-Q, \\
\frac{d}{d t}\left(a^{2} b\right)=-(n-1) \frac{T}{\pi \mu} a b K(b),
\end{gathered}
$$

where $S(t)$ denotes the cross-sectional area of $\Omega(t)$, and $K(\cdot)$ denotes the complete elliptic integral of the first kind (see (B 4) in Appendix B, with $\phi=\pi / 2$ ).

Howison \& Richardson considered an $(a, b)$ phase plane within which the univalency domain is $0 \leq b<1, a \geq 0$ (recall the comments at the end of $\S 2$ ); a solution trajectory $(a(t), b(t)$ ) reaching the univalency boundary $b=1$ is associated with formation of $3 / 2$-power cusps. Solution breakdown before extraction is inevitable when $T=0$, with $b\left(t^{*}\right)=1, a\left(t^{*}\right)>0$, for some positive "blow-up" time $t^{*}$. However solving (4.2) and (4.3) when $T>0$, they found that complete extraction of fluid always occurs with extraction time $t_{E}=S(0) / Q$ such that $a\left(t_{E}\right)=0, b\left(t_{E}\right)<1$. This naturally led them to consider the limiting case $T \rightarrow 0$ where, combining the previous two observations, cusps form in $\partial \Omega$ at time $t^{*}<t_{E}$, and persist until time $t_{E}$. This corresponds to a degenerate case of equation (4.3), where $K(b)$ on the right-hand side is singular as $b \uparrow 1$, but $T \rightarrow 0$ to counteract this effect. The net result is that $b(t)$ is "pinned" at 1 for $t>t^{*}$, while from (4.2), $a(t)$ evolves according to

$$
\frac{d}{d t}\left[\pi a^{2}(1+1 / n)\right]=-Q
$$

until $t=t_{E}$; this analysis also shows that ZST theory holds for $0<t<t^{*}$.

These solutions are not classical, because the free boundary is nonanalytic. There is an embryonic theory of such (so-called) "weak solutions" which is outlined in Appendix A (and which is similar in spirit to a theory advanced by Hohlov et al. [15] for the $T \rightarrow 0^{+}$limit of the Hele-Shaw problem with suction). In generality it demands too much technical machinery to describe here (in particular, its motivation requires more of the complex variable theory than we give) and we refer to [7] for the details. Our aim in this paper is to illustrate the dynamics of motion on the univalency boundary, and the sort of things that can happen. Indeed, given the tentative nature of the general "weak solution" theory, the direct approach of taking the limit $T \rightarrow 0$ which we adopt provides a useful verification of the theory, which is shown to be consistent in Appendix A. 
The weak solutions in the example of HR'95 are all of similarity type for $t^{*}<t<t_{E}$, since only the scaling parameter $a$ changes; the shape of the free boundary remains the same throughout. So, for instance, the solution for $n=2$ evolves as a shrinking cardioid for times $t>t^{*}$ in the limit $T \rightarrow 0$.

The precise nature of the singularity in (4.3) as $b \uparrow 1$ is determined by the asymptotic behaviour

$$
K(1-\epsilon) \sim-\frac{1}{2} \log (\epsilon / 8) \quad \text { as } \epsilon \rightarrow 0,
$$

(see for example [3]). This means that for small positive surface tension $T$, the quantity $(1-b)$ must be exponentially small in $1 / T$ before surface tension effects become important, a fact borne out in experiments [19], where 'almost-cusps', having radii of curvature which are exponentially small in the capillary number, can be observed.

\section{The cubic polynomial map}

\subsection{Real coefficients}

We now consider the ideas above applied to the more complicated case of a general cubic polynomial mapping function in the limit $T \rightarrow 0$. The ZST case of the analogous Hele-Shaw problem was solved by Huntingford in [18]. As explained, we expect the evolution of the free boundary $\partial \Omega(t)$ to follow ZST theory until the "blow-up time" $t^{*}$, at which point we relax the restriction on $\partial \Omega(t)$ to permit solutions with persistent cusps in the free boundary. The mapping function we consider is

$$
f(\zeta, t)=a(t)\left(\zeta+\frac{b(t)}{2} \zeta^{2}+\frac{c(t)}{3} \zeta^{3}\right)
$$

without loss of generality we assume the scaling factor $a$ to be real and positive for all time. By suitably rotating the co-ordinates in the initial domain $\Omega(0)$, the general case with both $b(0)$ and $c(0)$ complex may be reduced to an initial map $f(\zeta, 0)$ with just one complex coefficient. For simplicity we shall assume $b(0), c(0) \in \mathbb{R}$, which will then ensure $b(t)$ and $c(t)$ are also real for $t>0$; this is equivalent to the assumption that $\Omega(t)$ is symmetric about the $x$-axis. We return to the limitations of our assumption in $§ 5.2$. For this case, $(3.2),(3.3)$ and (3.5) yield the evolution equations

$$
\begin{aligned}
\frac{d}{d t}\left[a^{2}\left(1+\frac{b^{2}}{2}+\frac{c^{2}}{3}\right)\right] & =-\frac{Q}{\pi} \\
\frac{d}{d t}\left[a^{2} b\left(1+\frac{2 c}{3}\right)\right] & =-\frac{T}{2 \mu} G_{+}(0, t) a^{2} b\left(1+\frac{2}{3} c\right)-\frac{T}{3 \mu} G_{+}^{\prime}(0, t) a^{2} c \\
\frac{d}{d t}\left[a^{2} c\right] & =-\frac{T}{\mu} G_{+}(0, t) a^{2} c
\end{aligned}
$$

\subsubsection{The ZST case}

With $T=0$ these equations are valid until the time $t^{*}$ at which the map ceases to be univalent. As in [18] we must consider the domain $V$ in $(b, c)$-space for which $(5.1)$ is univalent ${ }^{4}$ on $|\zeta| \leq 1$, and find the phase trajectories of the system (5.3), (5.4) within $V$. Cowling \& Royster [4] determined this univalency domain, in the more general case of complex coefficients. For real coefficients, $V$ is symmetric about the $c$-axis (so we lose nothing by restricting attention to the right-half plane

\footnotetext{
4 Note, though, that we are actually considering the projection of a "univalency cylinder" in $(a, b, c)$ space, onto $a=1$, with (5.2) providing the extra information about the variation of $a$ with time.
} 


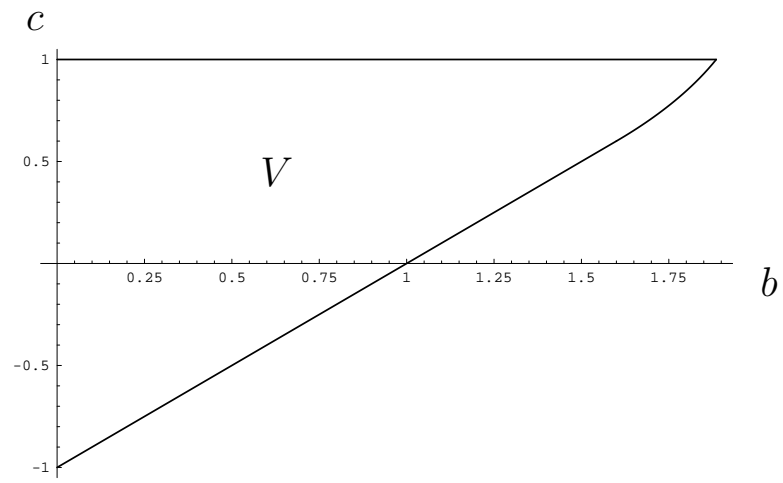

Figure 1. The univalency domain $V$ in $(b, c)$-space for the cubic polynomial mapping function.

$b>0)$, and is bounded in $b>0$ by the lines

$$
c=1, \quad b=1+c, \quad \text { and } \quad \frac{b^{2}}{4}+4\left(\frac{c}{3}-\frac{1}{2}\right)^{2}=1 .
$$

The domain $V$ is shown in Figure 1. Figure 2 shows free boundary "blow-up" shapes for various parameter values on $\partial V$. The line $b=1+c$ corresponds to formation of a single $3 / 2$-power cusp on $\partial \Omega(t)$, except for the isolated points $(0,-1)$ (where we have two $3 / 2$-power cusps, symmetrically placed about both axes), and $(8 / 5,3 / 5)$ (where we have a single $5 / 2$-power cusp). The line $c=1$ corresponds to two $3 / 2$-power cusps on $\partial \Omega$ (symmetrically placed about both axes when $b=0$ ), and the elliptical segment of $\partial V$ (which extends from $b=8 / 5$ to $b=4 \sqrt{2} / 3$ ) corresponds to loss of univalency in which the free boundary begins to overlap itself.

Equations (5.3) and (5.4) give the ZST phase paths within $V$ as the curves

$$
\frac{b}{c}\left(1+\frac{2 c}{3}\right)=\text { constant }=k,
$$

for various $k \in \mathbb{R}$. In contrast to the Hele-Shaw result of [18], we find no phase paths which meet $\partial V$ tangentially and then re-enter $V$; all ZST solutions blow up with the phase path hitting $\partial V$ obliquely (see Figure 4). A tangent phase path would be associated with the instantaneous formation of a cusp of $(4 N+1) / 2$-power type, which would immediately smooth (the phase path re-entering $V$ ) and the free boundary would become analytic again. Examples of such behaviour are known for Stokes flow [24] and the Hele-Shaw problem [15,16,18] involving transient 5/2-power cusps, but it does not occur here.

The ZST evolution is then fully determined, the general picture being that the free boundary of the fluid domain evolves through a series of smooth free boundary shapes towards a cusped or self-overlapping shape (depending on the initial shape $\partial \Omega(0)$ ) of the kind illustrated in Figure 2. We emphasise that in no instance can all the fluid be extracted from $\Omega(t)$ in the ZST case. The 
(1)

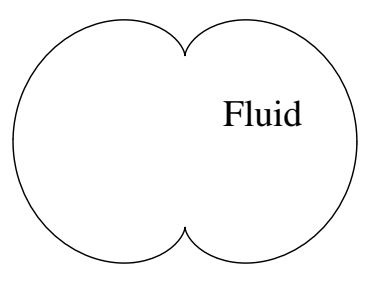

(3b)

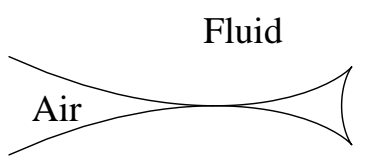

(2)

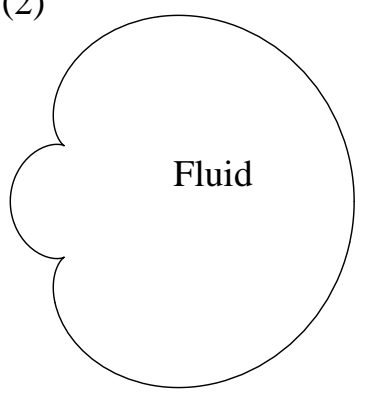

(4a)

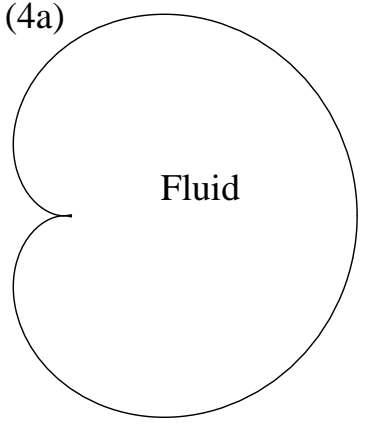

(5)

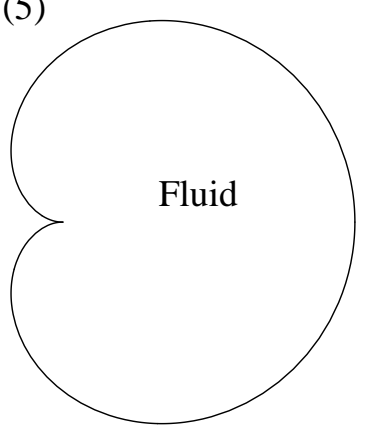

(6)

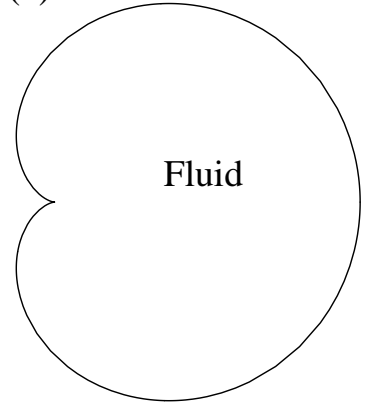

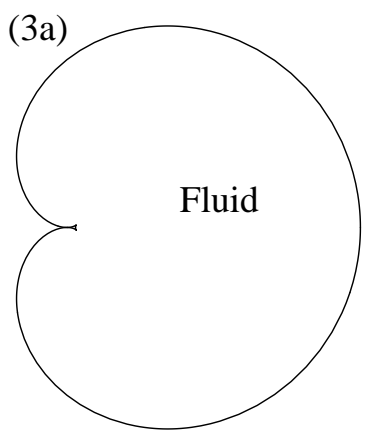

(4b)

Fluid

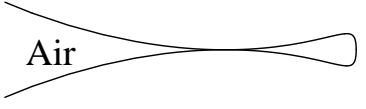

(7)

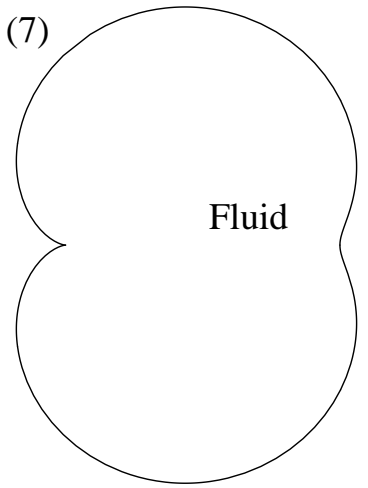

Figure 2. Free boundary shapes described by the map (5.1) for various points $(b, c)$ on the boundary $\partial V$ of the univalency domain. The values used are: $\left(b_{1}, c_{1}\right)=(0,1),\left(b_{2}, c_{2}\right)=(1,1),\left(b_{3}, c_{3}\right)=(4 \sqrt{2} / 3,1)$, $\left(b_{4}, c_{4}\right)=(1.8,0.8461),\left(b_{5}, c_{5}\right)=(8 / 5,3 / 5),\left(b_{6}, c_{6}\right)=(1,0)$, and $\left(b_{7}, c_{7}\right)=(1 / 5,-4 / 5)$. Pictures $(3 \mathrm{~b})$ and $(4 \mathrm{~b})$ are magnifications of the nonunivalent region, showing how the free boundary begins to overlap itself; the former case is cusped and self-overlapping, while the latter is smooth. The value $a=1$ was used to generate each picture, hence the shapes do not have equal areas.

phase-paths within $(b, c)$-space for this ZST solution are included in Figure 4, which clearly shows where the blow-up occurs.

\subsubsection{The case $T>0$ and its limit as $T \rightarrow 0^{+}$}

We now consider the effect of small positive surface tension, as we approach $\partial V$ along a phase path. Using the definition (2.5), we are able to find exact expressions for $G_{+}(0, t)$ and $G_{+}^{\prime}(0, t)$ in terms of elliptic integrals. These exact expressions are necessary if we wish to consider the problem with $O(1)$ surface tension (and may be used in the numerical solution of the $O(1)$ surface 
tension problem) but are not very illuminating for the present discussion of the limit $T \rightarrow 0$; hence we relegate the details to Appendix B. The main point to note is that they are singular only on the straight-line portions of $\partial V$ (i.e. those portions corresponding to blow-up via cusp formation rather than by overlapping) and so only in the neighbourhood of these lines are surface tension effects significant, justifying our earlier assumption that ZST theory is adequate for $t<t^{*}$. To find the phase paths near $\partial V$, we combine (5.3) and (5.4), writing $a^{2} c=A$, and $a^{2} b(1+2 c / 3)=B$, to give

$$
\frac{d B}{d A}=\frac{B}{2 A}+\frac{G_{+}^{\prime}(0, t)}{3 G_{+}(0, t)}
$$

and hence it is only the ratio of $G_{+}^{\prime}(0, t)$ to $G_{+}(0, t)$ that is important.

We need to consider two separate cases, according as to whether the ZST solution breaks down by reaching the boundary of the univalency domain at $c=1$, or at $b=1+c$ (refer forward to Figure 4). Consider first the class of solutions for which $c \uparrow 1$ within $\partial V$, along a ZST phase path. The crucial point to note is that once $c$ has reached a value close to 1 , it is 'trapped' near $c=1$ until either the solution blows up (with $c=1$ and attendant cusp formation, or with $c \simeq 1$, $b \simeq 4 \sqrt{2} / 3$ on the elliptical portion of $\partial V$, and self-overlapping of the free boundary), or until all fluid is extracted. The reason for this is simply that if $c$ decreased much below 1, ZST theory would again take over, forcing it back up towards $c=1$ on a ZST phase path. It follows that only $a$ and $b$ vary appreciably with time, and so $A \approx a^{2}, B \approx 5 a^{2} b / 3$. Essentially what we are doing is replacing one of the equations (5.2)-(5.4) with the requirement that the $T=0^{+}$phase-path must follow the univalency boundary $\partial V$ once it has reached it.

The results of Appendix B show that near $c=1$,

$$
\frac{G_{+}^{\prime}(0, t)}{G_{+}(0, t)} \approx-b \approx-\frac{3 B}{5 A}
$$

hence (5.7) becomes

$$
\frac{d B}{d A} \approx \frac{3 B}{10 A}
$$

giving $B=$ (const.) $\times A^{3 / 10}$, or, in terms of the mapping function parameters,

$$
b \approx(\text { const. }) \times a^{-7 / 5}, \quad c \approx 1 .
$$

Knowing that ZST theory will hold until $c \approx 1$, we may take $t^{*}$ (the ZST "blow up" time) to be zero without loss of generality and proceed from there, so that, in the limit $T \rightarrow 0, c(t) \equiv 1$ throughout the motion. Thus, from (5.2) and (5.8), the equations to be solved are

$$
a^{2}\left(\frac{4}{3}+\frac{b^{2}}{2}\right)=\frac{S(0)-Q t}{\pi}=a_{*}^{2}\left(\frac{4}{3}+\frac{b_{*}^{2}}{2}\right)-\frac{Q t}{\pi}
$$

and

$$
b=b_{*}\left(\frac{a_{*}}{a}\right)^{7 / 5},
$$

where we use $S(t)$ to denote the area of $\Omega(t)$, and $a_{*}, b_{*}$ denote the starting values of $a$ and $b\left(0 \leq b_{*}<4 \sqrt{2} / 3\right.$ and $c_{*}=1$, remember $)$. The right-hand side of (5.9) is simply a linearly decreasing function of time, reaching zero at the "extraction time" $t_{E}=S(0) / Q$. Substituting from (5.10) in (5.9) gives

$$
\mathcal{G}(b)-\mathcal{G}\left(b_{*}\right)=-\frac{6 Q t}{\pi a_{*}^{2} b_{*}^{10 / 7}}, \quad \text { where } \quad \mathcal{G}(b):=b^{-10 / 7}\left(8+3 b^{2}\right) .
$$

Now, $\mathcal{G}(b)$ is positive and monotone decreasing in $b$ on the range of interest (namely $0 \leq b \leq$ $4 \sqrt{2} / 3$ ), so (5.11) tells us that $b$ must be monotone increasing in $t$, from its starting value $b_{*}$. Hence the phase path must follow the line $c=1$ in this direction, ending either at time $t_{E}$, or 
when it reaches $b=4 \sqrt{2} / 3$. Complete extraction cannot occur in this regime, since (5.9) and (5.10) give the area of the fluid domain as

$$
S(t)=\pi\left(\frac{4}{3} a^{2}+\frac{b_{*}^{2} a_{*}^{14 / 5}}{2 a^{4 / 5}}\right),
$$

which is always positive. Hence we deduce that the phase path reaches $b=4 \sqrt{2} / 3$ before all the fluid has been extracted, and the solution breaks down with $\partial \Omega(t)$ beginning to overlap itself (Figure 2, (3a) and (3b)).

We now consider the case of solutions approaching the straight-line portion $b=1+c$ of $\partial V$ along a ZST phase path, observing, by the same argument as above, that a phase path is 'trapped' near this line once it is sufficiently close to it (Figure 4). We may thus eliminate either $b$ or $c$ in the ZST limit, and we choose to work with $b$ (so $c=b-1$ ). In this case, $A \approx a^{2}(b-1)$ and $B \approx a^{2} b(2 b+1) / 3$. The asymptotic evaluation of the ratio $G_{+}^{\prime}(0, t) / G_{+}(0, t)$ as the line $b=1+c$ is approached is performed in Appendix B. This limit is found to be nonuniform on the range $b \in(0,8 / 5)$, being equal to -2 everywhere except at the single point $b=0$. Thus for $b>0(5.7)$ becomes

$$
\frac{d B}{d A} \approx \frac{B}{2 A}-\frac{2}{3}
$$

which has solution

$$
B=-\frac{4 A}{3}+\lambda \sqrt{|A|},
$$

for some constant $\lambda$. We again take $t^{*}=0$ without loss of generality, and our initial conditions must satisfy $b_{*}=1+c_{*}$ (where now $0 \leq b_{*} \leq 8 / 5$ ). In terms of $a$ and $b$ then, we have

$$
\frac{a g(b)}{|b-1|^{1 / 2}}=\frac{a_{*} g\left(b_{*}\right)}{\left|b_{*}-1\right|^{1 / 2}} \equiv 3 \lambda,
$$

where $g(b):=2 b^{2}+5 b-4$, holding together with the mass conservation equation (5.2). After some rearrangement, and putting $c=b-1$ (since we remain on this part of the univalency boundary), (5.2) becomes

$$
\left(\frac{a}{a_{*}}\right)^{2} h(b)-h\left(b_{*}\right)=-\frac{6 Q t}{\pi a_{*}^{2}},
$$

for $h(b)$ defined by

$$
h(b):=5 b^{2}-4 b+8 \equiv 6\left(1+\frac{b^{2}}{2}+\frac{(b-1)^{2}}{3}\right) .
$$

Combining the previous two equations, eliminating the ratio $a / a_{*}$ between them, we finally arrive at an analogue of (5.11),

$$
|b-1| \frac{h(b)}{g(b)^{2}}-\left|b_{*}-1\right| \frac{h\left(b_{*}\right)}{g\left(b_{*}\right)^{2}}=-\frac{6 Q t}{\pi a_{*}^{2}} \frac{\left|b_{*}-1\right|}{g\left(b_{*}\right)^{2}} .
$$

Ignoring the two exceptional cases $b_{*}=1, \lambda=0$ for the moment (on our range of interest, $\lambda=0$ occurs if and only if $\left.b_{*}=b_{c}=(-5+\sqrt{57}) / 4\right)$, we see that the right-hand side of (5.12) is a monotone decreasing function of time, and so too must be the left-hand side, so that $F(b):=$ $|b-1| h(b) / g(b)^{2}$ decreases with time. The area of the fluid domain is given by

$$
S(t)=\frac{\pi a_{*}^{2} h\left(b_{*}\right)}{6} \frac{F(b)}{F\left(b_{*}\right)},
$$

so complete extraction occurs if and only if $F(b)$ falls to zero; this corresponds to extraction time $t_{E}=\pi a_{*}^{2} h\left(b_{*}\right) /(6 Q)$. A plot of $F(b)$ on $(0,8 / 5)$ is given in Figure $3(b=8 / 5$ is the point at which 

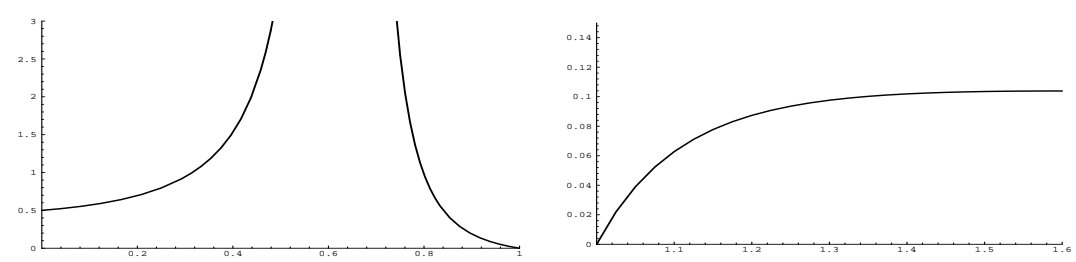

Figure 3. The function $F(b)$ governing evolution on the part $b=1+c$ of $\partial V$. The singularity occurs at $b=b_{c}=(-5+\sqrt{57}) / 4$. (Note the difference in scales between the two plots.

the form of $\partial V$ changes, the small elliptical portion of $\partial V$ for $8 / 5<b<4 \sqrt{2} / 3$ corresponding to blow up of solutions by overlapping of the free boundary). Important features to note are that:

- $F(b)$ vanishes only at $b=1$;

- $F(b)$ has a singularity at $b=b_{c}$, corresponding to a critical point in the phase diagram;

- $F(b)$ is monotone increasing (to infinity) on $\left(0, b_{c}\right)$, monotone decreasing (to zero) on $\left(b_{c}, 1\right)$, and monotone increasing on $(1,8 / 5)$;

- $F^{\prime}(b)=0$ at $b=8 / 5$, and only there, corresponding to the formation of the $5 / 2$-power cusp.

We must have $F(b)$ decreasing with $t$, hence for $b_{*} \in\left(b_{c}, 1\right)$ and $b_{*} \in(1,8 / 5)$, the phase path will approach the point $b=1, c=0$, with complete extraction occurring as we reach this point. By contrast, if $b_{*} \in\left(0, b_{c}\right)$ we must have the phase path approaching $b=0, c=-1$. Since $F(0)>0$, this point is reached before all the fluid has been extracted, but due to the symmetry of the phase diagram about the $c$-axis, we are forced to stay at this point. (For the moment we ignore the complications hinted at by the nonuniformity of the limit $G_{+}^{\prime}(0, t) / G_{+}(0, t)$ at this point.)

Recall now the comment in footnote (4), that we have actually been considering the projection of a univalency cylinder by suppressing the parameter $a$. We are thus in one of the special cases considered in HR'95; the subsequent evolution is of the 'similarity' type discussed there, with $b \equiv 0$, $c \equiv-1$, and the parameter $a$ changing in accordance with the corresponding mass conservation equation. The full phase diagram in the $(b, c)$-plane is given in Figure 4, with phase paths that are in some way 'special' represented by dashed lines. The bold arrows indicate the sense in which the phase paths 'turn around' as they hit $\partial V$.

It is now apparent that the 'exceptional cases' $b_{*}=1, b_{*}=b_{c}$ mentioned earlier are stable and unstable (respectively) critical points in the phase diagram, and thus also represent possible 'similarity' solutions of the kind studied in HR'95, the dotted phase path $c=0$ being exactly one of those solutions. Note that for this special solution, reaching $b=1$ no longer need be synonymous with total extraction, since the right-hand side of (5.12) is now identically zero; indeed, by the analysis of HR'95 we do remain a finite time at $(1,0)$ before extraction is complete. The points $(0,-1)$ and $(0,1)$ are also critical points, stable (but see $§ 5.2)$ and unstable respectively, and again, are members of the family of similarity solutions of HR'95. We may summarise our results as follows:

- Phase paths which hit $\partial V$ at $(1,0)$ or $(0,-1)$ terminate there and represent stable similarity solutions, since adjacent phase paths are also entering these points.

- Paths which hit $\partial V$ at $(0,1)$ and $\left(b_{c}, 1-b_{c}\right)$ terminate there and represent similarity solutions which are unstable, since neighbouring paths are diverging. 
Two 3/2-power cusps in $\partial \Omega$ along line $c=1$

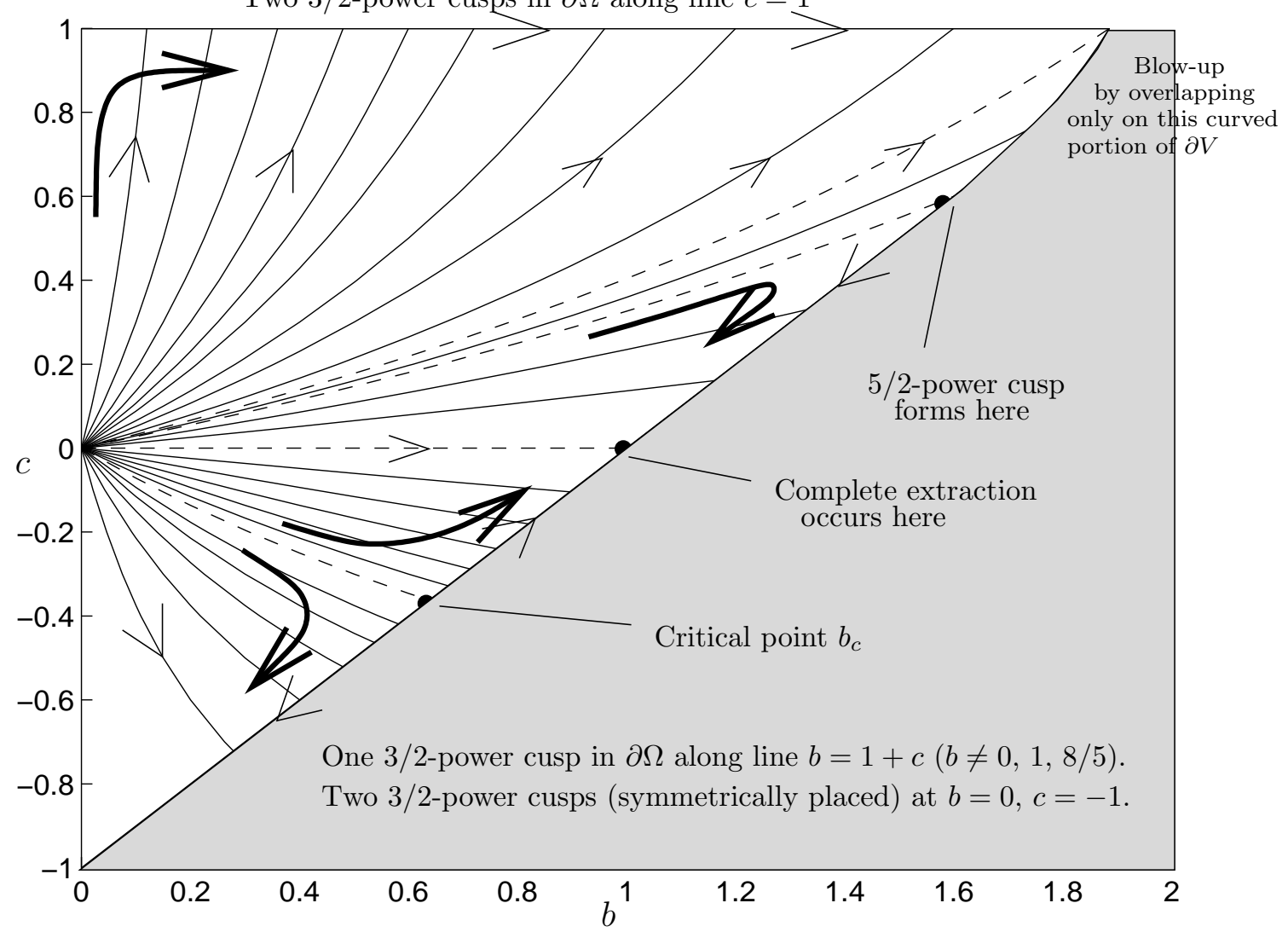

Figure 4. The univalency diagram (restricted to the right-half $(b, c)$-plane) for the $T \rightarrow 0$ solution with the cubic polynomial mapping function (5.1). The shaded region (outside $V$ ) corresponds to a nonunivalent map. The phase paths do not stop when they reach the boundary $\partial V$, but turn around and follow $\partial V$; this is indicated by the bold arrows.

- Paths for which $c_{*}=1, b_{*} \in(0,4 \sqrt{2} / 3)$ turn to the right and follow $\partial V$ along $c=1$, reaching $(4 \sqrt{2} / 3,1)$ before extraction is complete, at which point the free boundary begins to overlap itself. The present analysis then no longer applies, and the solution cannot be continued.

- Paths hitting the curved portion of $\partial V$ likewise represent self-overlapping fluid domains, and cannot be continued.

- Paths for which $c_{*}=b_{*}-1,1<b_{*}<8 / 5$ turn around and enter the point $(1,0)$; reaching this point is simultaneous with complete extraction.

- Ditto for $b_{c}<b_{*}<1$.

- Paths for which $c_{*}=b_{*}-1,0<b_{*}<b_{c}$ turn around and enter $(0,-1)$, reaching this point before extraction is complete; subsequent evolution is of 'similarity' type as discussed in HR' 95.

The 5/2-power cusp is an interesting borderline case, being the point of transition between ZST solutions which break down via formation of a 3/2-power cusp, and those which break down via overlapping of the free boundary. This path must still turn around and enter $(1,0)$; however, the fact that $F^{\prime}(8 / 5)=0$ (Figure 3) implies that this path 'only just makes it'. Geometrically, the $5 / 2$-power cusp immediately becomes a $3 / 2$-power cusp, which then persists. 
The existence of the point $b_{c}$ is also interesting. As we move along $\partial V$ from $(1,0)$ towards $(0,-1), \partial \Omega(t)$ evolves continuously from a cardioid (with a cusp on the left-hand side), to a fully symmetric shape having cusps on both sides. As is does so, a 'dimple' develops on the right-hand side (see (6) and (7) in Figure 2), which becomes more pronounced, eventually turning into the second cusp at the point $(0,-1)$ on $\partial V$. It is perhaps not surprising then that there is some critical point beyond which the 'dimple' is too large to disappear, and the ultimate shape has to have two cusps. If the dimple is small enough $\left(\right.$ i.e. $b_{*}>b_{c}$ ), then the ultimate shape will have just one cusp. For the solutions with $c_{*}=1$, however, the possible geometries are such that the two-cusp state is always unstable, and ultimate overlapping of the free boundary has to occur.

\subsection{Complex coefficients}

Recall our earlier comment, that the assumption of real coefficients in the mapping function (5.1) was equivalent to assuming symmetry of $\Omega(t)$ about the $x$-axis. The results obtained seem to have a remarkably rich structure nonetheless; however they are somewhat deceptive, as consideration of the case with complex coefficients reveals.

A little thought about the conclusions of $\S 5$ reveals an apparent contradiction: the point $(0,-1)$ in $(b, c)$-space is stated to be a stable equilibrium point, whilst the point $(0,1)$ is an unstable equilibrium point. But the two configurations are actually identical, one being a rotation through angle $\pi / 2$ of the other. In fact, the conclusions regarding the point $(0,-1)$ were a little suspect anyway, since we knew the limit $G_{+}^{\prime}(0, t) / G_{+}(0, t)$ to be nonuniform at this point, but the analysis away from this point did indicate that it should be a stable equilibrium.

In the preceding analysis, we have been considering a single, two-dimensional cross-section of what is actually a four-dimensional univalency domain $V_{4}$ in complex $(b, c)$-space. ${ }^{5}$ Determination of this domain is the subject of Cowling \& Royster's (henceforth C \& R) paper [4]. There, the authors note that the cross-section $\Im\{b\}=0$ of $V_{4}$ is symmetric about the planes $\Re\{b\}=0$ and $\Im\{c\}=0$, and so it may be assumed without loss of generality that $\Im\{b\}=0$ and $\Re\{b\} \geq 0$ (since this still generates all possible free boundary shapes, up to rotations and reflections). Writing $c=c_{1}+i c_{2}$ and taking $b \in \mathbb{R}^{+}$, their paper then determines this three-dimensional cross-section $\left(V_{3}\right.$, say) of $V_{4}$ for which the map (5.1) is univalent on the unit disc; however this domain is not simple, and is given in an implicit form which is difficult to use.

Before exploring the structure of this domain further, we consider the changes wrought in the evolution equations for the coefficients by allowing them to be complex. We assume still that $a \in \mathbb{R}^{+}$, but now write $b=b_{1}+i b_{2}$ and $c=c_{1}+i c_{2}$, for real $b_{1}, b_{2}, c_{1}, c_{2}$. The equations governing the ZST problem are found from (3.2), (3.3) and (3.5), and are

$$
\begin{aligned}
\frac{d}{d t}\left[a^{2}\left(1+\frac{|b|^{2}}{2}+\frac{|c|^{2}}{3}\right)\right] & =-\frac{Q}{\pi}, \\
a^{2}\left(b+\frac{2 c \bar{b}}{3}\right) & =\text { constant } \\
a^{2} c & =\text { constant }
\end{aligned}
$$

To obtain the phase paths in the four-dimensional $\left(b_{1}, b_{2}, c_{1}, c_{2}\right)$-space we must equate real and imaginary parts in these equations. The first is wholly real already, and in any case (as we have observed for the real coefficients case) is unnecessary for determination of the phase paths. The four real equations resulting from (5.15) and (5.14) are

$$
\begin{aligned}
& a^{2} c_{1}=k_{1}, \\
& a^{2} c_{2}=k_{2},
\end{aligned}
$$

5 In fact, bearing in mind the comments of footnote (4), the full univalency domain for the map (5.1) is a cylinder in five-dimensional space, but the dependence on the scaling parameter $a$ is of no consequence. 


$$
\begin{aligned}
& a^{2}\left(b_{1}+\frac{2}{3}\left(b_{1} c_{1}+b_{2} c_{2}\right)\right)=k_{3}, \\
& a^{2}\left(b_{2}+\frac{2}{3}\left(b_{1} c_{2}-b_{2} c_{1}\right)\right)=k_{4},
\end{aligned}
$$

which, as the four arbitrary constants $k_{1}$ to $k_{4}$ vary, give paths in $\left(b_{1}, b_{2}, c_{1}, c_{2}\right)$-space (after elimination of $a$ ). We now recall the statement of $\mathrm{C} \& \mathrm{R}$ that it is sufficient to consider the situation $b_{2}=0, b_{1}>0$. Suppose we seek such solutions to the above equations (5.16)-(5.19). The first two are unchanged, whilst the second two become

$$
\begin{aligned}
a^{2} b_{1}\left(1+\frac{2 c_{1}}{3}\right) & =k_{3}, \\
a^{2} b_{1} c_{2} & =k_{4} .
\end{aligned}
$$

Equations (5.16) and (5.17) give

$$
\frac{c_{1}}{c_{2}}=\text { constant }
$$

whilst (5.20) and (5.21) give

$$
\frac{1}{c_{2}}+\frac{2 c_{1}}{3 c_{2}}=\text { constant }
$$

which together imply that either both $c_{1}$ and $c_{2}$ must be constant, or else $c_{2}=0$. Supposing the first case, with $c_{2} \neq 0$, then to satisfy the equations we need both $a$ and $b_{1}$ to be constant also, in which case the mass conservation equation cannot hold (except in the trivial case $Q=0$ ). Hence we must have $c_{2}=0$, showing that the only family of solutions for which $b \in \mathbb{R}$ throughout the evolution, is that already found for which $c \in \mathbb{R}$ also.

The result of $\mathrm{C} \& \mathrm{R}$ essentially says that restricting attention to $V_{3}$ yields all possible free boundary shapes, the remainder of $V_{4}$ consisting of rotations and reflections of shapes which are contained within $V_{3}$. For a map with constant coefficients it is then sufficient to consider $V_{3}$, since any free boundary configuration can be generated by some point within $V_{3}$ provided the axes are suitably chosen. With time-dependent coefficients, we may choose axes such that the initial configuration $\Omega(0)$ is generated by a point of $V_{3}$; however the above shows that only if $c_{2}=0$ is the configuration for $t>0$ also generated by a point of $V_{3}$. Solution trajectories for $c_{2} \neq 0$ migrate to regions of $V_{4}$ outside $V_{3}$.

C \& R's observation is therefore of limited use, since the only family of solution trajectories lying wholly within the three-dimensional cross-section $V_{3} \subset V_{4}$ is the family of real solutions already studied - all other solution trajectories simply intersect $V_{3}$ at a single point. The full fourdimensional space $V_{4}$ is horribly difficult (if not impossible) to determine and study analytically. We consider instead whether we might find a three-dimensional solution family for the case in which $c$ is real, but $b$ is complex. Setting $c_{2}=0$ in equations (5.16)-(5.19) gives ${ }^{6}$

$$
\begin{gathered}
a^{2} c_{1}=k_{1}, \quad k_{2}=0 \\
a^{2} b_{1}\left(1+\frac{2 c_{1}}{3}\right)=k_{3}, \quad a^{2} b_{2}\left(1-\frac{2 c_{1}}{3}\right)=k_{4}
\end{gathered}
$$

since (5.17) has reduced to an identity, we are able to eliminate $a$ from these equations to find

${ }^{6}$ Note that this analysis also covers the case $c_{1}=0, c_{2} \neq 0$, since if we make the substitutions $B_{1}=\left(b_{1}+b_{2}\right) / \sqrt{2}, B_{2}=\left(b_{2}-b_{1}\right) / \sqrt{2}, \xi=\exp (-i \pi / 4) \zeta$ and $F=\exp (i \pi / 4) f$ we have

$$
F(\xi, t)=a \quad \xi+\frac{1}{2}\left(B_{1}+i B_{2}\right) \xi^{2}+\frac{c_{2}}{3} \xi^{3}
$$

so we get the same phase diagram in $\left(B_{1}, B_{2}, c_{2}\right)$-space. 
phase trajectories in $\left(b_{1}, b_{2}, c_{1}\right)$-space: these are determined by the two equations

$$
\frac{b_{1}}{c_{1}}\left(1+\frac{2 c_{1}}{3}\right)=\text { constant }, \quad \frac{b_{2}}{c_{1}}\left(1-\frac{2 c_{1}}{3}\right)=\text { constant }
$$

To get an idea of this three-dimensional cross-section $V_{*}$ of $V_{4}$, we consider simple two-dimensional cross-sections. The cross-section $b_{2}=0$ is the case already studied (the domain $V$ given by (5.5)). The cross-section $b_{1}=0$ corresponds to maps of the form

$$
\frac{f(\zeta, t)}{a}=\zeta+\frac{i b_{2}}{2} \zeta^{2}+\frac{c_{1}}{3} \zeta^{3}
$$

Making the substitutions $\zeta=-i \hat{\zeta}, c_{1}=-\hat{c_{1}}$ and $f(\zeta, t)=-i \hat{f}(\hat{\zeta}, t)$ we find that

$$
\frac{\hat{f}(\hat{\zeta}, t)}{a}=\hat{\zeta}+\frac{b_{2}}{2} \hat{\zeta}^{2}+\frac{\hat{c_{1}}}{3} \hat{\zeta}^{3},
$$

so the intersection of $V_{*}$ with this cross-section is exactly the domain $V$, but inverted with respect to $c_{1}$; call it $V_{\dagger}$. Likewise, we have a ZST solution family lying entirely within $V_{\dagger}$, with phase paths exactly as for the real coefficients case, but inverted with respect to $c_{1}$. The $T \rightarrow 0$ limit is also inferred from the earlier analysis.

The other two-dimensional cross-section of $V_{*}$ we can look at is $c_{1}=0$. This is particularly easy, the map now being

$$
\frac{f(\zeta, t)}{a}=\zeta+\frac{\left(b_{1}+i b_{2}\right)}{2} \zeta^{2}
$$

so that $f^{\prime}(\zeta, t)=0$ only if $\zeta=-1 /\left(b_{1}+i b_{2}\right)$, and the map is univalent on the disc

$$
b_{1}^{2}+b_{2}^{2} \leq 1
$$

A solution family again lies in this cross-section (which we call $V_{\ddagger}$ ), with solution trajectories which are straight lines

$$
\frac{b_{1}}{b_{2}}=\text { constant }
$$

as can be seen from (5.18) and (5.19) with $c_{1}=0=c_{2}$. All points on the univalency boundary are equivalent, in the sense that the free boundary shapes represented by the maps are just rotations of the same cardioid. The $T \rightarrow 0$ limit of this solution family is of the "similarity solution" type, with initial limaçons becoming cardioids (before all the fluid has been extracted) which then persist in a self-similar fashion until extraction is complete.

The schematic Figure 5 indicates how the three-dimensional domain $V_{*}$ fits together. Given the equivalence of the cross-sections $V$ and $V_{\dagger}$, we now see plainly the equivalence of the points $\{b=0, c=1\}$ and $\{b=0, c=-1\}$, and the arrows in Figure 5 show how this configuration destabilises.

The only point in our phase diagram (Figure 4) which is possibly still ambiguous is $b=1, c=0$, which is claimed to be stable. How do we know that this point does not 'destabilise' like the point $b=0, c=1$, by the phase paths moving onto $\partial V_{4} \not \subset \partial V$ ? Consider again the three-dimensional subset $V_{*} \subset V_{4}$, which we have seen contains a solution family. We know that phase paths of this family starting within $V_{*}$ move out to the boundary under the ZST evolution equations (5.22), and then move over the surface $\partial V_{*}$ under the influence of the $T=0^{+}$effect ( $c f$. the paths moving along the boundary $\partial V$ in Figure 4). From our experience with the real coefficients case, we know that a phase path can leave $\partial V_{*}$ through a critical point, and move onto other parts of $\partial V_{4}$. However, the point is that $V_{*}$ contains a solution family, and therefore the phase paths within $V_{*}$ (and on $\partial V_{*}$ ) must be topologically consistent, just as they are within $V$. It is easily checked (by sketching the phase paths on the surface of some suitable three-dimensional objecta plastic cup, in the authors' case!) that the only consistent possibility is for all phase paths to 

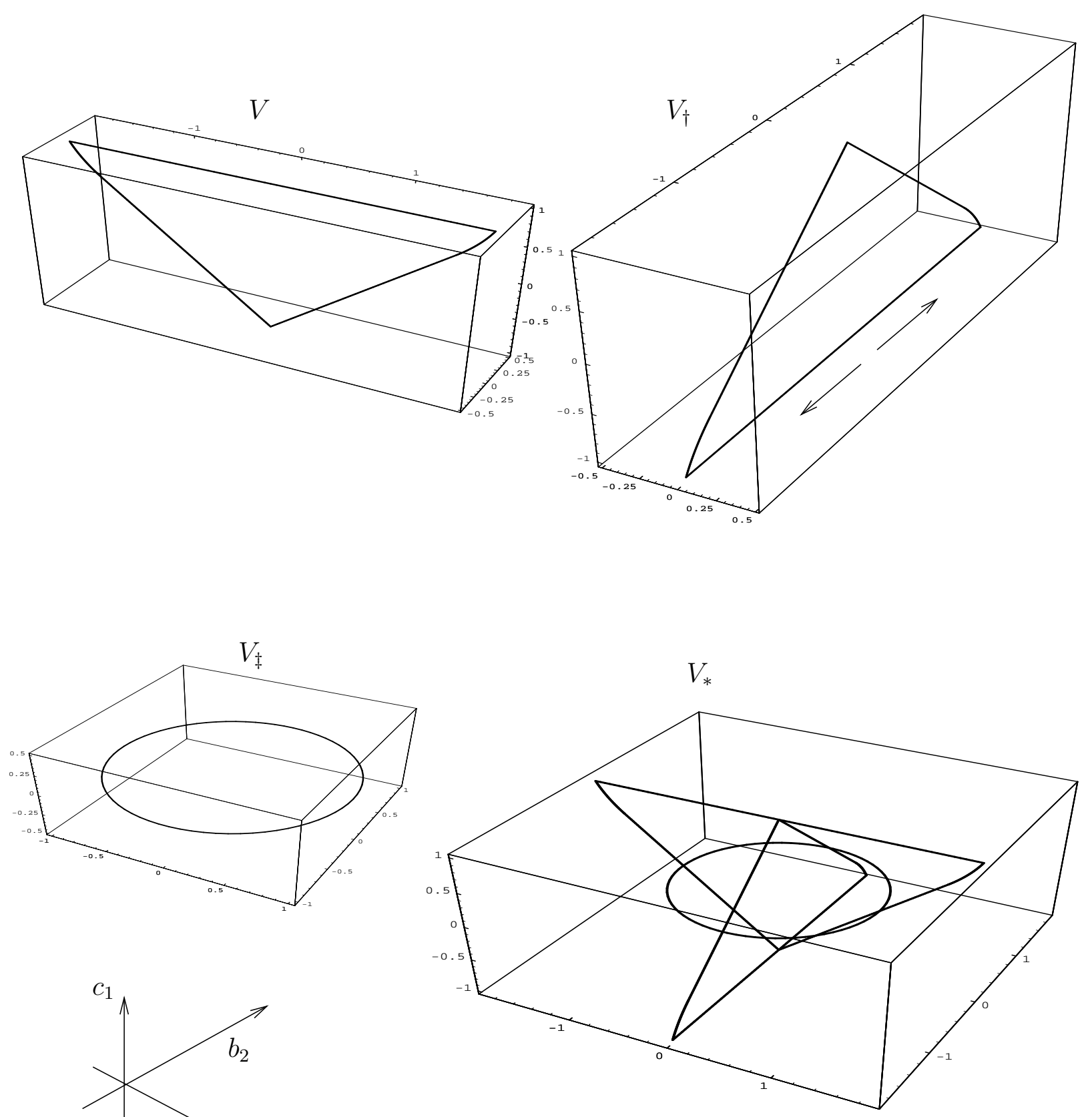

Figure 5. The three-dimensional univalency domain $V_{*} \subset V_{4}$, and its two-dimensional cross-sections $V$, $V_{\dagger}$ and $V_{\ddagger}$. The arrows on $V_{\dagger}$ indicate how the point $\{b=0, c=-1\}$ destabilises (cf. Figure 4 ). 
enter the ring $\left\{b_{1}^{2}+b_{2}^{2}=1, c_{1}=0\right\}$ on $\partial V_{*}$ (all points of which represent equivalent free boundary shapes). Thus, all such solution shapes are stable with respect to perturbations in $c_{1}$. Also, as observed in footnote 6 , there is an analogous family of solutions with $c_{1} \equiv 0$ and $c_{2} \neq 0$, which is contained within the same domain $V_{*}$ in $\left(B_{1}, B_{2}, c_{2}\right)$-space. In the same way then, solution shapes on $\left\{B_{1}^{2}+B_{2}^{2} \equiv b_{1}^{2}+b_{2}^{2}=1, c_{2}=0\right\}$ are stable to perturbations in $c_{2}$. Thus, the "cardioid" shapes with $c \equiv 0$ are the attractors of the cubic polynomial family we consider, and in this sense the stability of the point $b=1, c=0$ in Figure 4 is confirmed.

Our study is clearly not exhaustive, since we have considered only a limited subset $V_{*}$ of $V_{4}$, which contains a family of solutions of which the real coefficients case is a sub-family. In fact this sub-family appears twice within $V_{*}$, as we have seen (in $V$ and in $V_{\dagger}$ ) so there is considerable repetition even within this limited subset. $V_{*}$ does not contain all possible free boundary configurations. On the other hand, the cross-section $V_{3}\left(b_{2}=0\right)$ of $V_{4}$ is a minimal set of possible free boundary shapes if axes are chosen appropriately, but complex-parameter solutions do not lie wholly within this space. Evolution in time cannot be determined by studying $V_{3}$ then, unless the analysis is somehow modified to allow the co-ordinates within the fluid domain to rotate suitably in time - we do not consider this possibility.

\section{Conclusion}

We have discussed in detail the evolution of the weak solution for a cubic map ZST Stokes flow solution. We have confirmed that the weak solution is the usual ZST solution before blow-up, and we have analysed its progress around the boundary of the univalency domain at later times. For this particular map, the free boundary can have either one or two cusps when it is singular, and we have presented strong evidence (short of a full examination of the four-dimensional univalency domain, which does not appear feasible) that if all the fluid is extracted, the ultimate shape is generically a cardioid with one cusp, the two-cusped configurations being unstable or leading to self-overlapping. It is an interesting open question how many terminal cusps might be generic for a more complex map.

\section{Acknowledgements}

The authors are grateful for helpful conversations with Prof. J.R. King and Dr J.R. Ockendon.

\section{Appendix A The "weak solution" theory}

In this Appendix we briefly outline the "weak solution" concept advanced in [7], and its application to the cubic-mapping problem. Roughly speaking, from (2.3) and (2.5) one can see that naively setting $T=0$ in the govering equations gives the correct ZST limit as long as the free boundary is smooth $\left(f^{\prime}(\zeta) \neq 0\right.$ on $|\zeta|=1$, so $\left.t<t^{*}\right)$. However as $t \rightarrow t^{*}$ and a zero (or zeros) of $f^{\prime}(\zeta, t)$ approaches the unit circle (at $\zeta=\zeta^{*}$, say) the function $G_{+}(\zeta, t)$ becomes infinite as $(\zeta, t) \rightarrow$ $\left(\zeta^{*}, t^{*}\right)$. Thus we get "zero times infinity" in equation $(2.3): T \rightarrow 0$ is a singular limit. In [7] it is argued that if, in the ZST problem, cusps form at points $\left(\zeta_{j}^{*}, t_{j}^{*}\right)$ in $(\zeta, t)$-space, then in the limit $T \rightarrow 0$ we should make the substitution

$$
\frac{T}{2 \mu} G_{+}(\zeta, t) \mapsto \sum_{j} G_{j}(t)\left(\frac{\zeta_{j}^{*}+\zeta}{\zeta_{j}^{*}-\zeta}\right),
$$

where the functions $G_{j}(t)$ must be determined (by the usual singularity-matching) but are real and satisfy

$$
G_{j}(t) \equiv 0 \quad \text { for } t<t_{j}^{*}
$$


Thus the $G_{j}(t)$ act as "switches" for the singularities at $\zeta_{j}^{*}$ which appear at time $t_{j}^{*}$. Looking back at the definition of $G_{+}(\zeta, t)$ in $(2.5)$, one can see that (A 1$)$ is a reasonable ansatz, since as the singularity formation times $t_{j}^{*}$ are approached the major contributions to the integral come from the points $\zeta=\zeta_{j}^{*}$. Matching the singular behaviour in equation (2.3) at the new singularities immediately gives the result $f^{\prime}\left(\zeta_{j}^{*}, t\right)=0$ for $t>t_{j}^{*}$, that is, the cusps persist. In this general theory, more than one "breakdown time" $t^{*}$ is permitted because in the weak formulation the solution does not break down at time $t^{*}$ but continues, with non-analytic free boundary. Note that the $\zeta_{j}^{*}$ may themselves be functions of $t$, i.e. the cusps may move along the free boundary (and do, in our example).

Thus in the $T \rightarrow 0$ limit the governing equation (2.3) is replaced by its equivalent with the substitution (A 1), and (as mentioned in $\S 2$ ) solutions to the model may be obtained by matching the singular behaviour on both sides of the new equation (for our example the only singularity other than those at the cusps is at $\zeta=0$ ). However we may circumvent this singularity-matching analysis by observing that in line with the substitution (A 1) we must have

$$
\frac{T}{2 \mu} G_{+}^{\prime}(\zeta, t) \mapsto 2 \sum_{j} \frac{G_{j}(t) \zeta_{j}^{*}}{\left(\zeta_{j}^{*}-\zeta\right)^{2}}
$$

Thus instead of evaluating the limit $G_{+}^{\prime}(\zeta, t) / G_{+}(0, t)$ as the univalency boundary is neared (which was the direct approach in $\S 5$; see equation $(5.7))$, we need to determine

$$
L:=\frac{2 \sum\left(G_{j}(t) / \zeta_{j}^{*}\right)}{\sum G_{j}(t)}
$$

for $t>t^{*}$ in the two breakdown cases.

\section{Case (I): Breakdown by approaching the line $c=1$}

In this case two cusps form simultaneously. In the notation introduced above we have (by symmetry)

$$
\zeta_{1}^{*}=\zeta^{*}, \quad \zeta_{2}^{*}=\bar{\zeta}^{*}, \quad t_{1}^{*}=t_{2}^{*}=t^{*}, \quad G_{1}(t)=G_{2}(t)=G(t),
$$

where $\zeta^{*}=\left(-b+i \sqrt{b^{2}-4}\right) / 2$. Thus since $\left|\zeta^{*}\right|=1$ the definition (A 2) gives

$$
L=2 \Re\left(\zeta^{*}\right)=-b,
$$

precisely as obtained (more painfully) in Appendix B (equation (B 5)).

\section{Case (II): Breakdown by approaching the line $b=1+c$}

In this case (for $c \neq-1$ ) we have a single cusp forming at the point $\zeta^{*}=-1$. Thus it is trivial to see that

$$
L=-2
$$

in this case, again in agreement with the results of Appendix B (equation (B 6)).

\section{Appendix B Asymptotic analysis near the univalency boundary}

In this Appendix we give the exact and asymptotic expressions for the functions $G_{+}(0, t)$ and $G_{+}^{\prime}(0, t)$. Time-dependence is suppressed henceforth, for brevity.

Using the definition (2.5) we find

$$
G_{+}(0) \equiv \frac{1}{\pi a} \int_{0}^{\pi} \frac{d \theta}{\left[b^{2}+(c-1)^{2}+2 b(1+c) \cos \theta+4 c \cos ^{2} \theta\right]^{1 / 2}},
$$




$$
\text { and } \quad G_{+}^{\prime}(0) \equiv \frac{2}{\pi a} \int_{0}^{\pi} \frac{\cos \theta d \theta}{\left[b^{2}+(c-1)^{2}+2 b(1+c) \cos \theta+4 c \cos ^{2} \theta\right]^{1 / 2}},
$$

the forms of which functions change as we cross the curve $b^{2}=4 c$ in $V$ (according to whether the denominator has real or complex roots as a function of $\cos \theta) ; G_{+}(0)$ itself is continuous across this curve, however. In $b^{2}<4 c$ we use formula 3.145.2 in Gradshteyn \& Ryzhik [10] (henceforth $\mathrm{G} \& \mathrm{R}$ ), and also the asymptotic result

$$
K(1-\epsilon) \sim-\frac{1}{2} \log (\epsilon / 8) \sim-\frac{1}{2} \log \epsilon \quad \text { as } \epsilon \rightarrow 0,
$$

where $K(\cdot)$ is the complete elliptic integral of the first kind. We find that

$$
\begin{aligned}
G_{+}(0) & =\frac{2 K\left(k_{1}\right)}{\pi a \sqrt{(c+1)^{2}-b^{2}}}, \quad \text { where } \quad k_{1}^{2}:=\frac{4 c-b^{2}}{(c+1)^{2}-b^{2}}, \\
& \sim \frac{-2}{\pi a \sqrt{4-b^{2}}} \log (1-c) \quad \text { as } c \uparrow 1,
\end{aligned}
$$

$c=1$ being the only singularity within this part of $V$. In $b^{2}>4 c$ we need formulae 3.147 .6 and 3.147.4 of $\mathrm{G} \& \mathrm{R}$ (in regions $c>0, c<0$ respectively) together with (B 1) to deduce that

$$
\begin{aligned}
G_{+}(0) & =\frac{2 K\left(k_{2}\right)}{\pi a\left(\sqrt{b^{2}-4 c}+(1-c)\right)} & \text { where } \quad k_{2}^{2}:=\frac{4(1-c) \sqrt{b^{2}-4 c}}{\left[\sqrt{b^{2}-4 c}+(1-c)\right]^{2}} \\
& \sim \frac{-1}{\pi a(1-c)} \log (1+c-b) & \text { as }(1+c-b) \downarrow 0,
\end{aligned}
$$

$b=1+c$ now being the only line of singularities within $V$. Explicit formulae for $G_{+}^{\prime}(0)$ are much more complicated; in $b^{2}<4 c$ we find

$$
\begin{gathered}
G_{+}^{\prime}(0)=\frac{-b(1+c) K\left(k_{1}\right)}{\pi a c \sqrt{(1+c)^{2}-b^{2}}}+\frac{2}{\sqrt{c}}\left\{E\left(k_{1}\right) F\left(\psi, k_{1}^{\prime}\right)+K\left(k_{1}\right)\left(E\left(\psi, k_{1}^{\prime}\right)-F\left(\psi, k_{1}^{\prime}\right)\right)\right\}, \\
\text { where }\left(k_{1}^{\prime}\right)^{2}=1-k_{1}^{2}, \quad \psi=\sin ^{-1}\left(\frac{b}{2 \sqrt{c}}\right) ;
\end{gathered}
$$

here $k_{1}$ is as previously defined, $E(\cdot), E(\cdot, \cdot)$ denote the complete and incomplete (respectively) elliptic integrals of the second kind, and $F(\cdot, \cdot)$ denotes the incomplete elliptic integral of the first kind (so $K(\cdot) \equiv F(\pi / 2, \cdot)$ ). The key formulae used in finding this expression were 259.07 and 410.02 in Byrd \& Friedman [3] (henceforth B \& F), along with various properties of elliptic integrals and Jacobian elliptic functions, all of which may be found in B \& F.

In $b^{2}>4 c$ we find

$$
\begin{array}{r}
G_{+}^{\prime}(0)=\frac{4}{\pi a\left(\sqrt{b^{2}-4 c}+(1-c)\right)}\left\{A K\left(k_{2}\right)-(1+A) \Pi\left(\frac{2}{1-A}, k_{2}\right)\right\}, \\
\text { where } A=\frac{1}{4 c}\left(-b(1+c)+(1-c) \sqrt{b^{2}-4 c}\right) ;
\end{array}
$$

again, $k_{2}$ is as previously defined, and $\Pi(\cdot, \cdot)$ denotes the complete elliptic integral of the third kind. In finding this expression the formulae used were G \& R 3.148.6 and 3.148.4 (in regions $c>0$ and $c<0$ respectively).

In using these two books, care was necessary to account for slight differences in definitions. Likewise, when carrying out numerical checks on the analysis, care was needed due to different inbuilt definitions in the software package Mathematica. The above assumes the definitions:

$$
\begin{aligned}
& F(\phi, k)=\int_{0}^{\phi} \frac{d \theta}{\sqrt{1-k^{2} \sin ^{2} \theta}}=\int_{0}^{\sin \phi} \frac{d x}{\sqrt{\left(1-x^{2}\right)\left(1-k^{2} x^{2}\right)}}, \\
& E(\phi, k)=\int_{0}^{\phi} \sqrt{1-k^{2} \sin ^{2} \theta} d \theta=\int_{0}^{\sin \phi} \frac{\sqrt{1-k^{2} x^{2}}}{\sqrt{1-x^{2}}} d x,
\end{aligned}
$$




$$
\begin{aligned}
\Pi\left(\alpha^{2}, k\right) & =\int_{0}^{\pi / 2} \frac{d \theta}{\left(1-\alpha^{2} \sin ^{2} \theta\right) \sqrt{1-k^{2} \sin ^{2} \theta}} \\
& =\int_{0}^{1} \frac{d x}{\left(1-\alpha^{2} x^{2}\right) \sqrt{\left(1-x^{2}\right)\left(1-k^{2} x^{2}\right)}} .
\end{aligned}
$$

The results of $\S 5$ require the asymptotic evaluation of the ratio $G_{+}^{\prime}(0) / G_{+}(0)$ near each of the lines $c=1$ and $b=1+c$. This is not too bad for the case $c \uparrow 1$, and fairly nasty for the case $b \downarrow(1+c)$; we give only brief details.

In $b^{2}<4 c$ results of B \& F $\S \S 111-112$ are used, together with (B 1) above, to deduce that as $c \uparrow 1$, the term in curly brackets in $G_{+}^{\prime}(0)$ (B 2) is everywhere negligible compared to the first term. Hence we see that the asymptotic behaviour here is

$$
\frac{G_{+}^{\prime}(0)}{G_{+}(0)} \sim-b .
$$

To study the behaviour of $G_{+}^{\prime}(0)$ as $b \uparrow(1+c)$ in the region $b^{2}>4 c$ we must consider the cases $c>0$ and $c<0$ separately, since these give different types of behaviour in (B 3). We write $\epsilon=1+c-b$ and eliminate $b$ to work with $c$ and $\epsilon$, so that letting $\epsilon \rightarrow 0$ corresponds to approaching the univalency boundary $\partial V$. We also define the auxiliary parameter $\delta:=\epsilon^{2} /\left(4(1-c)^{2}\right)$; this will always be small since we do not consider the elliptical part of $\partial V$ corresponding to blow-up via overlapping of the free boundary, so $c$ lies in the range $-1<c<3 / 5$. We find:

$$
\begin{array}{r}
A=-1-2 \delta+\cdots, \quad-(1+A)=2 \delta+\cdots, \\
k_{2}^{2}=1-\delta\left(\frac{1+c}{1-c}\right)^{2}+\cdots, \quad \alpha^{2} \equiv \frac{2}{1-A}=1-\delta+\cdots .
\end{array}
$$

We know the asymptotic behaviour of the first term in curly brackets in $G_{+}^{\prime}(0)$ (B 3), from (B 1). The term outside, multiplying the curly bracket, is also straightforward. Hence we only need to find the behaviour of the second term within curly brackets, which to lowest order is

$$
-(1+A) \Pi\left(\frac{2}{1-A}, k_{2}\right) \sim 2 \delta \Pi\left(\alpha^{2}, k_{2}\right) .
$$

Suppose first that $c \in(0,3 / 5)$. Then by the above expressions, $0<\alpha^{2}<k_{2}^{2}<1$, and so according to the classifications of B \& F (p. 223) we have a case II elliptic integral of the third kind (a circular case). ${ }^{7}$ Formula 412.01 in B \& F thus applies, giving the result in terms of the Heuman Lambda function. Results from $\S 150$ of the book may then be used to arrive at the approximation

$$
2 \delta \Pi\left(\alpha^{2}, k_{2}\right)=\frac{1-c}{\sqrt{c}}\left(\frac{\pi}{2}-\sin ^{-1}\left(\frac{1-c}{1+c}\right)\right)+O(\delta \log \delta),
$$

which gives excellent agreement when checked numerically. This term will thus be everywhere negligible compared to the first term in the curly brackets $\left(K\left(k_{2}\right)\right.$ being singular as $\left.k_{2} \rightarrow 1\right)$, hence we get the approximation

$$
G_{+}^{\prime}(0) \sim \frac{2}{\pi a(1-c)} \log (1+c-b) .
$$

It follows that for $c$ in this parameter range we will have

$$
\frac{G_{+}^{\prime}(0)}{G_{+}(0)} \sim-2,
$$

as we approach the boundary.

For $c \in(-1,0)$ (still using (B 3)) we have $0<k_{2}^{2}<\alpha^{2}<1$, which is a case III elliptic integral

7 The case $c=0$ is the special case $\alpha^{2}=k^{2}$, and provides a check on the analysis in both regions $c>0$, $c<0$. 
of the third kind (a hyperbolic case). Thus formula 414.01 of B \& F applies, and it is relatively easy to see that

$$
2 \delta \Pi\left(\alpha^{2}, k_{2}\right) \simeq \frac{1-c}{\sqrt{-c}} K\left(k_{2}\right) Z\left(\beta, k_{2}\right) \quad \text { for } \quad \beta=\sin ^{-1}\left(\frac{\alpha}{k_{2}}\right)=\frac{\pi}{2}-\frac{2 \sqrt{-c \delta}}{1-c}+\cdots,
$$

where $Z(\cdot, \cdot)$ denotes the Jacobi Zeta function (discussed in $\S 140$ of B \& F). Then

$$
G_{+}^{\prime}(0) \sim \frac{-2 K\left(k_{2}\right)}{\pi a(1-c)}\left(1-\frac{1-c}{\sqrt{-c}} Z\left(\beta, k_{2}\right)\right)
$$

so that

$$
\frac{G_{+}^{\prime}(0)}{2 G_{+}(0)} \sim-1+\frac{1-c}{\sqrt{-c}} Z\left(\beta, k_{2}\right) .
$$

Results of $\S 140$ and $\S 100$ in B \& F show that for small $\delta$,

$$
Z\left(\beta, k_{2}\right) \sim \frac{1}{K\left(k_{2}\right)} \log \left(\frac{2 \sqrt{-c}}{1+c}+\left(1-\frac{4 c}{(1+c)^{2}}\right)^{1 / 2}\right) .
$$

Note that $c=0$ is not a problem point, despite the factor $1 / \sqrt{-c}$ in the above, because for small $c$ we may expand the logarithmic term appearing in the expression for $Z\left(\beta, k_{2}\right)$. The only problem is at $c=-1$; away from this point we can see that

$$
\frac{G_{+}^{\prime}(0)}{G_{+}(0)} \sim-2
$$

Near $c=-1$, the function $Z\left(\beta, k_{2}\right)$ will no longer be negligible according to the above. Here we have

$$
\frac{G_{+}^{\prime}(0)}{2 G_{+}(0)} \sim-1+2\left(1+\frac{\log \epsilon}{\log (1+c)}\right)^{-1}=-1+2\left(1+\frac{\log \epsilon}{\log (\epsilon+b)}\right)^{-1},
$$

for $c$ close to -1 (or, $b$ small and positive). So, for instance, if we take $b=\lambda \epsilon$ for some order one quantity $\lambda$ we will have

$$
\frac{G_{+}^{\prime}(0)}{G_{+}(0)} \rightarrow 0 \quad \text { as } \epsilon \rightarrow 0 .
$$

In particular, this will be the case as we approach the univalency boundary along the phase path $b \equiv 0$. We thus have a nonuniform limit, with

$$
\frac{G_{+}^{\prime}(0)}{G_{+}(0)} \rightarrow-2 \quad \text { as } \epsilon \rightarrow 0
$$

everywhere except $c=-1$ (or $b=0)$; at this point the limit is zero. 


\section{References}

[1] Antanovskit, L.K. Influence of surfactants on a creeping free-boundary flow induced by two counterrotating horizontal thin cylinders. Eur. J. Mech. B (Fluids), 13 (1), 73-92 (1994).

[2] Buckmaster, J.D. Pointed bubbles in slow viscous flow. J. Fl. Mech. 55, 385-400 (1972).

[3] Byrd, P.F., Friedman, M.D. Handbook of Elliptic Integrals for Engineers and Scientists. SpringerVerlag (1971).

[4] Cowling, V.F., Royster, W.C. Domains of variability for univalent polynomials. Proc. Amer. Math. Soc. 19, 767-772 (1968).

[5] Crowdy, D.G., Tanveer, S. A theory of exact solutions for plane viscous blobs. J. Nonlinear Sci. 8, 261-279 (1998).

[6] Cummings, L.J., Howison, S.D., King, J.R. Conserved quantities in Stokes flow with free surfaces. Phys. Fluids 9 (3), 477-480 (1997).

[7] Cummings, L.J., Howison, S.D., King, J.R. Two-dimensional Stokes and Hele-Shaw flows with free surfaces. To appear in Europ. J. Appl. Math. (1999).

[8] Duren, P.L. Univalent Functions. Springer-Verlag (1983).

[9] Garabedian, P.R. Free boundary flows of a viscous liquid. Comm. Pure ES Appl. Math. XIX (4), 421-434 (1966).

[10] Gradshteyn, I.S., Ryzhik, I.M. Table of Integrals, Series and Products (corrected and enlarged edition). Academic Press, inc. (1980).

[11] Hopper, R.W. Coalescence of two equal cylinders: Exact results for creeping viscous plane flow driven by capillarity. J. Am. Ceram. Soc. (Comm.) 67, C 262-264. Errata ibid. 68 C 138 (1985).

[12] Hopper, R.W. Plane Stokes flow driven by capillarity on a free surface. J. Fl. Mech. 213, 349-375 (1990).

[13] Hopper, R.W. Stokes flow of a cylinder and a half-space driven by capillarity. J. Fl. Mech. 243, 171-181 (1992).

[14] Hohlov, Yu.E., Howison, S.D. On the classification of solutions to the zero surface tension model for Hele-Shaw free boundary flows. Quart. Appl. Math. 51, 777-789 (1993).

[15] Hohlov, Yu.E., Howison, S.D., Huntingford, C., Ockendon, J.R., Lacey, A.A. A model for non-smooth free boundaries in Hele-Shaw flows. Q. Jl. Mech. Appl. Math. 47 (1), 107-128 (1994).

[16] Howison, S.D. Cusp development in Hele-Shaw flow with a free surface. SIAM J. Appl. Math. 46 (1), 20-26 (1986).

[17] Howison, S.D., Richardson, S. Cusp development in free boundaries, and two-dimensional slow viscous flows. Europ. J. Appl. Math. 6 (5), 441-454 (1995).

[18] Huntingford, C. An exact solution to the one-phase zero surface-tension Hele-Shaw free boundary problem. Computers Math. Applic. 29 (10), 45-50 (1995).

[19] Jeong, J., Moffatt, H.K. Free-surface cusps associated with flow at low Reynolds number. J. Fl. Mech. 241, 1-22 (1992).

[20] Joseph, D.D., Nelson, J., Renardy, M., Renardy, Y. Two-dimensional cusped interfaces. J. Fl. Mech. 223, 383-409 (1991).

[21] Richardson, S. Two-dimensional bubbles in slow viscous flow. J. Fl. Mech. 33, 475-493 (1968).

[22] Richardson, S. Two-dimensional bubbles in slow viscous flow. Part 2. J. Fl. Mech. 58, 115-127 (1973).

[23] Richardson, S. Two-dimensional slow viscous flows with time-dependent free boundaries driven by surface tension. Europ. J. Appl. Math. 3, 193-207 (1992).

[24] Richardson, S. Two-dimensional Stokes flows with time-dependent free boundaries driven by surface tension. Europ. J. Appl. Math. 8, 311-329 (1997).

[25] Tanveer, S. Evolution of Hele-Shaw interface for small surface tension. Phil. Trans. Roy. Soc. Lond. A 343, 155-204 (1993).

[26] Tanveer, S., Vasconcelos, G.L. Time-evolving bubbles in two-dimensional Stokes flow. J. Fl. Mech. 301, 325-344 (1995). 